\title{
Factors Affecting Trial Without Catheter for First Spontaneous Acute Urinary Retention
}

\author{
Punit Mahadik, Surya Prakash Vaddi, Chandra-Mohan Godala, V. Vijaya Kumar Reddy, Venkat Krishna Sambar \\ Department of Urology \& Renal Transplantation, Narayana Medical College, Nellore, India
}

\begin{abstract}
Purpose: To find the association of trial without catheter (TWOC) outcome for first spontaneous acute urinary retention (AUR) in benign prostatic obstruction with age, prior lower urinary tract symptoms (LUTS), retention volume at catheterization (RV), and size of prostate.

Methods: Our prospective observational analytical (interventional) study enrolled 77 cases of spontaneous AUR over 24 months. After clinical evaluation, digital rectal examination, and transabdominal ultrasonography, all patients were catheterized per urethra and their RV was recorded. TWOC was administered after 2 or 3 doses of $0.4 \mathrm{mg}$ tamsulosin-oral absorption control system and after 48-72 hours had passed. A successful endpoint was defined as a maximum flow-rate, $>5 \mathrm{~mL} / \mathrm{sec}$; voided volume, $>100$ $\mathrm{mL}$; postvoid residue, $<200 \mathrm{~mL}$; and voiding within 6 hours of catheter removal. Data obtained from 58 patients were analyzed after excluding the cases lost to follow-up and secondary exclusion. Age, RV, duration of LUTS, and prostate volume on examination and ultrasonography (PUSG) were recorded and statistically analyzed. Prostate-specific antigen levels were obtained on follow-up and cases of cancer, as seen on transrectal ultrasound-guided biopsy, were secondarily excluded.

Results: The patients had a mean age of $65.89 \pm 8.67$ years. Prior LUTS was seen in 35 patients ( $2.07 \pm 2.91$ months). The mean PUSG and RV were $46.81 \pm 20.58 \mathrm{~mL}$ and $854.8 \pm 36.26 \mathrm{~mL}$, respectively. Thirty patients underwent a successful TWOC; a mean age of $63.13 \pm 8.58$ years (mean \pm standard deviation; unpaired $\mathrm{t}$-test; $\mathrm{P}=0.0053$ ) and a PUSG of $\leq 45 \mathrm{~mL}$ (Pearson chi-square test; $\mathrm{P}=0.0427$ ) were significantly associated with a successful outcome.

Conclusion: There is a significant association between TWOC outcome, age $(\mathrm{P}=0.0053)$, and PUSG $(\mathrm{P}=0.0427)$.
\end{abstract}

Keywords: Urinary retention; Tamsulosin; Urination disorders

\section{INTRODUCTION}

Acute urinary retention (AUR) is one of the most significant long-term outcomes resulting from benign prostatic hyperplasia (BPH). The painful inability to void is often an unexpected, important, and feared event from both an economic standpoint and the viewpoint of the patient. The need for an emergent hospital visit, catheterization, follow-up visits, attempt at catheter removal, and eventual management is a tiring and time-consuming process. As a result of our recently increased understanding of the incidence, risk factors, and systemic effects of AUR, its management has changed. AUR in elderly men is often thought to be due to $\mathrm{BPH}$ but age also contributes to its incidence. The primary focus of the initial evaluation and diagnostic testing should be differentiating spontaneous from precipitated AUR, which is important from both a clinical and a prognostic viewpoint. In the past, the first approach was early surgery, which is safer in the absence of an indwelling catheter [1]. The second approach was long-term catheterization (transurethral, suprapubic, or intermittent), with obvious disadvantages. The third approach was trial without catheter (TWOC) in an ambulant or in-patient setting and usually with medical therapy, for which reported success rates have currently improved. A successful trial is followed by either transurethral resection of the prostate
Corresponding author: Punit Mahadik

Department of Urology \& Renal Transplantation, Narayana Medical College, 203 Pavani Elegance Near Apollo Hospital, Nellore 524001, Andhra Pradesh, India

Tel: +919494641319 / Fax: +918612331763 / E-mail: punit_mahadik@rediff.com Submitted: July 30, 2013 / Accepted after revision: September 9, 2013
This is an Open Access article distributed under the terms of the Creative Commons Attribution Non-Commercial License (http://creativecommons.org/licenses/by-nc/3.0/) which permits unrestricted non-commercial use, distribution, and reproduction in any medium, provided the original work is properly cited. 
(TURP) or medical therapy with outpatient follow-up. Failure requires recatheterization and reassessment of future management options, such as surgery, a second trial, or long-term catheterization. There are advantages to both the patient and the health care system of a successful TWOC, which has become a standard practice worldwide [2]. Selective TWOC may be imperative to minimize unnecessary suffering from AUR in less easily accessible health care facilities like ours and will reduce the total cost of treatment. Despite ongoing research and clinical studies, however, many unanswered questions remain, such as which patients may need to be prepared for eventual TURP. If success can be predicted before a trial of voiding, men who are likely to fail can be appropriately counseled and the exercise of a trial can be avoided in those most likely to fail. The obvious challenge is standardization of a protocol for selective TWOC in our health care setting. Alfuzosin is a proven drug for use in TWOC, whereas some evidence has also emerged for tamsulo$\sin$.

\section{MATERIALS AND METHODS}

The study enrolled 77 cases over 24 months at our medical college hospital. The inclusion criteria were as follows: all consecutive patients attending the urology clinics with AUR (painful, not impending/painless) and with a provisional clinical diagnosis of benign prostatic obstruction (BPO) due to $\mathrm{BPH}$. Detailed clinical history and examination including digital rectal examination (DRE) were performed and the results recorded by use of a predesigned form. Ultrasonography (USG) of the abdomenpelvis was done on presentation. All patients were catheterized via the urethra, residual urine volume (RV) was recorded, and urine was collected for routine and microscopic examination. Patients were discharged from the outpatient department with the advice to take tamsulosin-oral absorption control system 0.4 mg OD and to return to the outpatient department for the trial after 2 days. TWOC was administered in the hospital and was considered successful if the patient voided within 6 hours of catheter removal with a voided volume $>100 \mathrm{~mL}$ and postvoid residual volume $<200 \mathrm{~mL}$ on the basis of currently prevalent criteria in the literature. Those failing to void in 6 hours were managed by admission or recatheterization. Exclusion criteria were as follows: diagnosis other than BPO, residual urine $>1,200 \mathrm{~mL}$, any neurological history, or recent use of $5 a$-reductase inhibitors, a1-blockers, tricyclic antidepressants, anticholinergics, sympathomimetics, or first-generation anti-
Table 1. Distribution of the clinical features of the study population

\begin{tabular}{|c|c|c|c|}
\hline Characteristic & $\begin{array}{l}\text { TWOC } \\
\text { success }\end{array}$ & TWOC failure & Total \\
\hline Age (yr) & $63.13 \pm 8.58$ & $68.86 \pm 7.89$ & $65.89 \pm 8.67$ \\
\hline $\begin{array}{l}\text { Retention volume at } \\
\text { catheterization }(\mathrm{mL})\end{array}$ & $850.7 \pm 49.85$ & $859.3 \pm 53.75$ & $854.8 \pm 36.26$ \\
\hline PSA levels (ng/mL) & $8.30 \pm 2.24$ & $6.50 \pm 1.29$ & $7.43 \pm 1.31$ \\
\hline $\begin{array}{l}\text { DRE (prostate size } \\
\text { clinical grade) }\end{array}$ & $\mathrm{n}=30$ & $\mathrm{n}=28$ & $\mathrm{n}=58$ \\
\hline Grade 1 & $13(68.42)$ & $6(31.58)$ & $19(100)$ \\
\hline Grade 2 & $13(44.83)$ & $16(55.17)$ & $29(100)$ \\
\hline Grade 3 & $4(40.00)$ & $6(60.00)$ & $10(100)$ \\
\hline $\begin{array}{l}\text { Prostate volume on } \\
\text { USG }(\mathrm{mL})\end{array}$ & $\mathrm{n}=29$ & $\mathrm{n}=28$ & $\mathrm{n}=57$ \\
\hline$\leq 45$ & $18(62.07)$ & $11(37.93)$ & $29(100)$ \\
\hline$>45$ & $11(39.29)$ & $17(60.71)$ & $28(100)$ \\
\hline $\begin{array}{l}\text { Duration of prior } \\
\text { LUTS }\end{array}$ & $\mathrm{n}=17$ & $\mathrm{n}=18$ & $\mathrm{n}=35$ \\
\hline Less than 3 months & $14(56.00)$ & $11(44.00)$ & $25(100)$ \\
\hline More than 3 months & $3(30.00)$ & $7(70.00)$ & $10(100)$ \\
\hline
\end{tabular}

Values are presented as mean \pm standard error of the mean or number (\%).

TWOC, trial without catheter; PSA, prostate-specific antigen; DRE, digital rectal examination; USG, ultrasonography; LUTS, lower urinary tract symptoms.

histamines.

Written informed consent was obtained. We did not administer AUA scores to our patients for two reasons: most were educated less than standard 5th (a relative contraindication) and, moreover, such patients fail to completely comprehend even the native language AUA score when explained by the examiner. The cases underwent prostate-specific antigen (PSA) testing on follow-up at 6 weeks to eliminate falsely raised PSA due to retention or catheterization. Subsequently, those whose PSA values remained high $(>4 \mathrm{ng} / \mathrm{mL}$ ) underwent prostate biopsy. Among them, any patient positive for cancer on transrectal ultrasound-guided biopsy was secondarily excluded.

\section{RESULTS}

A total of 58 patients were analyzed, of whom 30 had a successful TWOC. Six patients were excluded for prostate cancer and one for vesical calculus (missed on the USG). Attrition of 12 cases occurred. Table 1 depicts the salient findings. All continu- 
ous data were checked for skewness and kurtosis of the distribution. Unpaired t-test was used for age and RV, chi-square test for duration of prior lower urinary tract symptoms (LUTS) and prostate volume on USG, and chi-square test for trend for size on DRE.

The age range of the patients was 45 to 87 years, and 30 (51.72\%) were older than 65 years. The mean age was less in the group with a successful TWOC. The difference between the means of the two groups was $5.72 \pm 2.16$ years (mean \pm standard error of the mean $[\mathrm{SEM}] ; \mathrm{P}=0.0053 ; 95 \%$ confidence interval [CI], 1.38 to 10.06). The sensitivity of the cutoff of 63 years to predict outcome was $43.33 \%$ (specificity, $78.57 \%$ ).

Thirty-five patients (60.35\%) reported having prior LUTS. Three reported storage-predominant LUTS, and the maximum duration was up to 12 months before AUR, with a mean ( \pm standard deviation [SD]) of $2.07 \pm 2.91$ months. Fifty-six percent with a duration of less than 3 months were successful, compared with $30 \%$ among those with a duration of longer than 3 months (risk ratio [RR], 1.867; 95\% $\mathrm{CI}, 0.6807$ to 5.119; $\mathrm{P}=0.0822$ ). The sensitivity of this cutoff to predict outcome was $82.35 \%$ and specificity was $38.89 \%$. Concerning prostate size on the DRE, $68.42 \%, 44.83 \%$, and $40 \%$ of patients with grade 1,2 , and 3 , respectively, had a successful trial. A trend was seen of decreasing success with increasing grade $(\mathrm{P}=0.0979)$. Concerning prostate size (volume) on USG (transabdominal), the data were abnormally distributed, with one outlier value of $150 \mathrm{~mL}$ and the next lower value being $85 \mathrm{~mL}$ (range, 10 to $150 \mathrm{~mL}$ ). Therefore, to maintain a normal distribution and to keep the analysis logically meaningful and statistically significant, the one outlier was excluded from further analysis. Thus, for these 57 cases, the mean $( \pm S D)$ value was $46.81 \pm 20.58 \mathrm{~mL}$ with a median of 45 $\mathrm{mL}$. A total of $62.07 \%$ and $39.29 \%$ of men with prostate volume less than or greater than $45 \mathrm{~mL}$, respectively, had a successful trial (RR, 1.580; 95\% CI, 0.9194 to 2.715; $\mathrm{P}=0.0427$ ). The sensitivity of this cutoff to predict outcome was $62.07 \%$ and specificity was $60.71 \%$. For RV, the difference between means was $8.619 \pm 73.31 \mathrm{~mL}( \pm \mathrm{SEM})(\mathrm{P}=0.4534)$.

\section{DISCUSSION}

Management of this urological emergency is still not standardized because of a lack of guidelines on this important subject.

\section{Age}

In the large Reten survey [2], median age was 70 years, com- pared with 66.5 years in our study. For TWOC at a median of 5 days ( $41.3 \%$ for $<3$ days), age of $<70$ years was associated with a successful TWOC $(\mathrm{P}<0.001)$. Our mean $( \pm \mathrm{SD})$ age of successful TWOC patients was $63.13 \pm 8.58$ years $(68.86 \pm 7.89$ years for failed). The $95 \%$ CI was 1.389 to 10.06 ( $\mathrm{P}=0.0053)$. Our cutoff for success was 7 years younger, which may partly have been due to the following:

1) The younger age (by 3.5 years) of our study population; the percentage in our study was $17.2 \%$ compared with the world survey in which $33.3 \%$ of men were aged above 74 years.

2) The survey looks impressive but also included precipitated AUR cases.

3) A diagnosis of BPH was based purely on history and DRE alone; no USG findings were reported.

4) Some older cases might have had smaller prostates than suggested by DRE and hence might have more likely succeeded. A median of 5 days was used for the trial, which might have improved the success in those in whom the TWOC was performed without an $\alpha$-blocker.

5) The results for age were not reported separately for those given an a-blocker, probably because the study was not designed for this.

The ALFAUR trial [3] showed that age over 65 years was negatively associated with success $(\mathrm{P}<0.001)$, a conclusion very similar to ours. McNeill et al. [4] reported age as a factor affecting outcome in a trial with alfuzosin. In that study, the mean age of the 47 patients who failed the TWOC was 73.1 years, which is very similar to our study. Pandit et al. [5], with a variable trial period of 3 to 15 days, also reported age to be higher for unsuccessful TWOC, but that study was observational and used three different $\alpha$-blockers and finasteride over a wide range of days. Ko et al. [6], using Fisher linear discriminant analysis, showed that age and RV had an $81.6 \%$ hit ratio for success. Further, based on this finding, a prospective trial showed improved success of $76.2 \%$ from $30.3 \%$. By use of urodynamic assessments, Djavan et al. [7] found age $>75$ years to be associated with failure. Thus, the importance of older age in association with failure is evident.

\section{Duration of Prior LUTS}

Our study found a marginally significant correlation $(\mathrm{P}=0.0822$; RR, 1.867; 95\% CI, 0.680 to 5.119) between duration of LUTS less than 3 months and successful TWOC. Some urologists consider the presence of previous LUTS before AUR to be a rel- 
ative indication for TURP [1]. In the largest international survey to date, $63 \%$ reported any LUTS previously [2]. Compared with precipitated AUR, spontaneous AUR was characterized by significantly severe LUTS (22.3\% vs. 31.7\%; $\mathrm{P}<0.001)$. Duration of prior LUTS was not reported, however, as in most of the literature. Unpublished data from the Alf-one study [8] indicate a worsening score with time as a predictor of AUR, thus indirectly implicating the duration of LUTS.

\section{Prostate Size on DRE}

We found a marginally significant correlation $(\mathrm{P}=0.0979)$ of a linear trend for success as gland size decreased. In our study, $68.42 \%$ ( 13 cases) of grade $1,44.83 \%$ (13 cases) of grade 2 , and $40 \%$ ( 4 cases) of grade 3 cases were successful. This is in accordance with the 5 studies available in the literature discussed below.

It is noteworthy that in the Reten study [2], estimates of prostate size were based on DRE alone and prostate size was categorized into three groups ( $<30 \mathrm{~g}, 30-50 \mathrm{~g}$, and $>50 \mathrm{~g}$ ). Overall, $43.1 \%$ had prostate size of $>50 \mathrm{~g}$. Including those who underwent a first TWOC with an $\alpha$-blocker (tamsulosin for 16.5\%), univariate regression analysis for trial at a median of 5 days ( $41.3 \%$ for $<3$ days) showed that size of $<50 \mathrm{~g}$ was associated with a successful TWOC $(\mathrm{P}<0.001)$. Multivariate regression analysis confirmed this (odds ratio, 1.92; 95\% CI, 1.52 to 2.42; $\mathrm{P}<0.001$ ). In a prospective audit of 100 patients who underwent TWOC without $\alpha$-blockers, volume on USG was not considered [9]. Success was more likely in patients with smaller glands $(\mathrm{P}=0.004)$. Another multivariate analysis of 72 men who underwent TWOC while taking $\alpha$-blockers reported similar conclusions [10]. In a prospective study of 40 men with 2-day TWOC by Kumar et al. [11], mean size was $15.9(10 \pm 30)$ and $27.5(10 \pm 50) \mathrm{g}(\mathrm{P}<0.006)$ for the success and failure groups, respectively. Eight were already taking an $\alpha$-blocker. Note that prostate size was assessed (subjectively) by a DRE by a single investigator only and not by USG. In a prospective randomized trial by McNeill et al. [4] of alfuzosin sustained-release $5 \mathrm{mg}$ twice daily in 40 men for initial spontaneous AUR, only DRE estimates for size categorized as small $(<20 \mathrm{~g})$, medium (21-50 $\mathrm{g}$ ), and large ( $\geq 51 \mathrm{~g}$ ) were used; these size categories were associated with a statistically significant trend for long-term outcome in a multiple Cox regression. The rough estimate of size by DRE appeared to be precise enough, with DRE estimates and transrectal ultrasound volumes correlating significantly, but they underestimated size by $25 \%$ to $55 \%$ for a volume over
$40 \mathrm{~mL}$. It is often of more interest to determine the degree of enlargement rather than to make a precise estimate [12].

\section{Prostate Size (Volume) on USG (Transabdominal)}

We reported a significant correlation $(\mathrm{P}=0.0427)$ for prostate size, which agrees with the world literature. In our study, $62.07 \%$ with a prostate volume of less than $45 \mathrm{~mL}$ had a successful trial, and $39.29 \%$ of cases with a prostate volume of more than $45 \mathrm{~mL}$ had a successful trial. The relative risk was 1.580 (95\% CI, 0.9194 to 2.715 ). The sensitivity of this cutoff to predict outcome was $62.07 \%$ and the specificity was 60.71 . Bhomi and Bhattachan [13] reported a prostate size of $40 \mathrm{~g}$ on transabdominal ultrasound to predict outcome, with a specificity of $73 \%$ and a sensitivity of $84 \%$. That study had 64 cases of first spontaneous AUR who underwent TWOC with tamsulosin $0.4 \mathrm{mg}$ for 3 days. The slightly higher number of cases than ours seems to have improved the sensitivity.

In a prospective study by Mariappan et al. [14], 54 men with a mean $( \pm \mathrm{SD})$ volume of $69.7 \pm 36.3 \mathrm{~mL}$ and spontaneous AUR underwent a trial with a-blocker for 2 weeks and had volume measured by standard transrectal USG. Pooled t-test was used to compare the means, which showed a smaller prostate size in the successful group (mean difference, $22 \mathrm{~mL}$; 95\% CI, 2.7 to 41.3; $\mathrm{P}=0.03$ ). In another retrospective analysis of 57 patients with first spontaneous TWOC given over 3 to 15 days [5], prostate volume was higher for patients with unsuccessful TWOC. By contrast, in one study from China by Hua et al. [15], the results of an a-blocker trial in 36 men showed that efficacy of treatment was not influenced by the volume of the prostate. The sample size of 36 appears to have been too small. As mentioned previously, intravesical protrusion has shown encouraging correlation with outcomes.

\section{Urinary Retention Volume}

We did not find a significant correlation $(\mathrm{P}=0.4534)$ but the volume complies with the trend detected in three previous studies, which found a cutoff volume of 900 to $1,000 \mathrm{~mL}[10,16,17]$. In one study, Djavan et al. [7] concluded from urodynamic assessment in patients with AUR that RV $>1 \mathrm{~L}$ was associated with failure. Lim et al. [18] reported on 79 men with first spontaneous AUR, for which RV $>800 \mathrm{~mL}$ correlated with failure, but no statistical significance was reported. A similar finding without statistical significance was reported by Zeif et al. [9] for about 100 cases with median RV of $750 \mathrm{~mL}$. Higher volumes (mean, $916 \mathrm{~mL}$ ) resulted in lower success rates. The authors suggested 
their limited value in predicting outcomes and recommend giving the "benefit of the doubt" and offering TWOC regardless of a large RV. In a similar vein, an RV of $800 \mathrm{~mL}$ was shown to predict outcome (specificity, $82 \%$; sensitivity, $64 \%$ ) by Bhomi and Bhattachan [13]. In that study, the authors created cutoff values from 64 cases of first spontaneous AUR given a trial on tamsulosin $0.4 \mathrm{mg}$ for 3 days but statistical significance was not achieved. Our results corroborate these studies.

In the Reten survey [2], univariate regression analysis showed that $\mathrm{RV}$ of $<1,000 \mathrm{~mL}$ was associated with a successful TWOC $(\mathrm{P}<0.001)$, and the results of the multivariate regression confirmed this. The survey looks impressive but included precipitated AUR cases also; furthermore, no USG findings were reported. A median of 5 days ( $41.3 \%$ for $<3$ days) was used for the trial, which might have improved the success of those who underwent TWOC without an $\alpha$-blocker. The results for RV and a-blocker use were not reported separately because the study was not designed for this. In the ALFAUR study [3], higher mean volumes, i.e., $983.8 \pm 270.9( \pm \mathrm{SD}) \mathrm{mL}$, might have contributed toward attaining statistical significance. Our volumes were approximately $100 \mathrm{~mL}$ less. Furthermore, the mean size, which does affect the outcome of a trial, in the success and failure groups is anybody's guess because they were not reported. In the study, an RV of $>1,000 \mathrm{~mL}$ adversely influenced the successful voiding rate in the logistic and multivariate regressions. In a prospective cohort [19] with a 3-day trial and a mean RV of $580.53 \pm 226.31( \pm S D)$, the cutoff volume associated with failure by use of the chi-square test was less than $700 \mathrm{~mL}(\mathrm{P}=$ 0.001).The mean RV for successful and failed cases were $547 \mathrm{~mL}$ and $706 \mathrm{~mL}$, respectively $(\mathrm{P}=0.02)$. This is less than our mean volumes of $850.7 \pm 49.85 \mathrm{~mL}( \pm$ SEM) for successful cases and probably explains the lack of statistical significance in our study. Similarly, in a study of 72 men by Daly et al. [10], the multivariate analysis showed an $\mathrm{RV}$ of $\geq 1,000 \mathrm{~mL}$ to have significance. This is more than our mean volume of $859.3 \pm 284 \mathrm{~mL}$ for failed cases and probably explains the lack of statistical significance in our study. The prospective study [11] of 40 men with spontaneous AUR and 2-day TWOC mentioned earlier reported mean RVs of $814 \mathrm{~mL}$ and $1,062 \mathrm{~mL}(\mathrm{P}=0.09)$ for the success and failure groups, respectively, compared with our difference of 8.619 $\mathrm{mL}$, which thus explains the lack of statistical significance in our study. In a randomized double-blind trial, 20 of 72 men with spontaneous AUR underwent single intermittent catheterization with administration of tamsulosin $0.4 \mathrm{mg}$ [20]. Multivariate analyses revealed RV to be the only significant determi- nant $(\mathrm{P}<0.01)$. The cutoff with $100 \%$ sensitivity was $500 \mathrm{~mL}$. With values of $750 \mathrm{~mL}$ and $1,000 \mathrm{~mL}$, the sensitivity was $76 \%$ and $18.3 \%$, respectively. Our mean values of over $800 \mathrm{~mL}$ would thus have poor specificity, which explains the lack of statistical significance in our study. The variability in postvoid residual volume is well known and RV probably follows suit. It is likely that better, specifically designed studies are needed to settle the issue of the significance of RV.

In conclusion, there was a significant association between TWOC outcome and age $(\mathrm{P}=0.0053)$ and volume of the prostate on ultrasound $(\mathrm{P}=0.0427)$ in patients with spontaneous first AUR with $\mathrm{BPO}$ given tamsulosin.

\section{CONFLICT OF INTEREST}

No potential conflict of interest relevant to this article was reported.

\section{REFERENCES}

1. Pickard R, Emberton M, Neal DE. The management of men with acute urinary retention. National Prostatectomy Audit Steering Group. Br J Urol 1998;81:712-20.

2. Fitzpatrick JM, Desgrandchamps F, Adjali K, Gomez Guerra L, Hong SJ, El Khalid S, et al. Management of acute urinary retention: a worldwide survey of 6074 men with benign prostatic hyperplasia. BJU Int 2012;109:88-95.

3. McNeill SA, Hargreave TB; Members of the Alfaur Study Group. Alfuzosin once daily facilitates return to voiding in patients in acute urinary retention. J Urol 2004;171(6 Pt 1):2316-20.

4. McNeill AS, Rizvi S, Byrne DJ. Prostate size influences the outcome after presenting with acute urinary retention. BJU Int 2004;94:55962.

5. Pandit RK, Agrawal CS, Chalise PR, Sapkota G. Retrospective analysis of management of patients presenting with acute urinary retention due to benign prostatic hyperplasia: a hospital based study. Kathmandu Univ Med J (KUMJ) 2008;6:448-52.

6. Ko YH, Kim JW, Kang SG, Jang HA, Kang SH, Park HS, et al. The efficacy of in-and-out catheterization as a way of trial without catheterization strategy for treatment of acute urinary retention induced by benign prostate hyperplasia: variables predicting success outcome. Neurourol Urodyn 2012;31:460-4.

7. Djavan B, Madersbacher S, Klingler C, Marberger M. Urodynamic assessment of patients with acute urinary retention: is treatment failure after prostatectomy predictable? J Urol 1997;158:1829-33. 
8. Fitzpatrick JM, Kirby RS. Management of acute urinary retention. BJU Int 2006;97 Suppl 2:16-20.

9. Zeif H, Wallace DM, Subramonian K. Predictors of successful trial without catheter in acute urinary retention. Br J Med Surg Urol 2010;3:5-10

10. Daly P, Connolly S, Rogers E, Sweeney P. Management outcome of acute urinary retention: model of prediction. Urol Int 2009;83:39-43.

11. Kumar V, Marr C, Bhuvangiri A, Irwin P. A prospective study of conservatively managed acute urinary retention: prostate size matters. BJU Int 2000;86:816-9.

12. Roehrborn CG, Sech S, Montoya J, Rhodes T, Girman CJ. Interexaminer reliability and validity of a three-dimensional model to assess prostate volume by digital rectal examination. Urology 2001; 57:1087-92.

13. Bhomi KK, Bhattachan CL. Factors predicting the success of a trial without catheter in acute urinary retention secondary to benign prostatic hyperplasia. Nepal Med Coll J 2011;13:178-81.

14. Mariappan P, Brown DJ, McNeill AS. Intravesical prostatic protrusion is better than prostate volume in predicting the outcome of trial without catheter in white men presenting with acute urinary retention: a prospective clinical study. J Urol 2007;178:573-7.

15. Hua LX, Wu HF, Sui YG, Chen SG, Xu ZQ, Zhang W, et al. Tamsulosin in the treatment of benign prostatic hyperplasia patients with acute urinary retention. Zhonghua Nan Ke Xue 2003;9:510-1.

16. Hastie KJ, Dickinson AJ, Ahmad R, Moisey CU. Acute retention of urine: is trial without catheter justified? J R Coll Surg Edinb 1990; 35:225-7.

17. Djavan B, Shariat S, Omar M, Roehrborn CG, Marberger M. Does prolonged catheter drainage improve the chance of recovering voluntary voiding after acute urinary retention (AUR) [abstract]. Eur Urol 1998;33(Suppl 1):110. Abstract no. 437.

18. Lim KB, Wong MY, Foo KT. The outcome of trial off catheter after acute retention of urine. Ann Acad Med Singapore 1999;28:516-8.

19. Li YK, Leung CS, Hui TL, Chiu LH. Acute urinary retention: how useful is an ambulatory care protocol? Hong Kong J Emerg Med 2009;16:134-40.

20. Drasa K. Acute urinary retention due to Benign Prostatic Hyperplasia and single intermittent catheterisation. Eur Urol Suppl 2009; 8:614-5. 